\title{
The role of thermal pressure in jet launching
}

\author{
Noam Soker \\ Department of Physics, Technion, Haifa 32000, Israel
}

\begin{abstract}
I present and discuss a unified scheme for jet launching that is based on stochastic dissipation of the accretion disk kinetic energy, mainly via shock waves. In this scheme, termed thermally-launched jet model, the kinetic energy of the accreted mass is transferred to internal energy, e.g., heat or magnetic energy. The internal energy accelerates a small fraction of the accreted mass to high speeds and form jets. For example, thermal energy forms a pressure gradient that accelerates the gas. A second acceleration stage is possible wherein the primary outflow stretches magnetic field lines. The field lines then reconnect and accelerate small amount of mass to very high speeds. This double-stage acceleration process might form highly relativistic jets from black holes and neutron stars. The model predicts that detail analysis of accreting brown dwarfs that launch jets will show the mass accretion rate to be $\dot{M}_{\mathrm{BD}} \gtrsim 10^{-9}-10^{-8} M_{\odot} \mathrm{yr}^{-1}$, which is higher than present claims in the literature.
\end{abstract}

Keywords. ISM: jets and outflows, stars: formation.

\section{Introduction}

In many popular models for the formation of astrophysical massive jets (to distinguish from low density hot-plasma jets from radio pulsars) magnetic fields play a dominate role in accelerating the jet's material from the accretion disk. Most models in young stellar objects (YSOs) are based on the operation of large scale magnetic fields driving the flow from the disk. In the "X-wind mechanism" introduced by Shu et al. $(1988,1991)$ the jets are launched from a narrow region in the magnetopause of the stellar field. A different model, although also using open radial magnetic field lines, is based on an outflow from an extended disk region, and does not rely on the stellar magnetic field (Ferreira \& Pelletier 1993, 1995; Wardle \& Königl 1993; Königl \& Pudritz 2000; Shu et al. 2000; Ferreira 2002; Krasnopolsky et al. 2003; Ferreira \& Casse 2004).

Other MHD simulations show that the high post-shock thermal pressure might accelerate gas and form jets and/or winds, e.g., as in the accretion around a black hole (BH) simulations performed by De Villiers et al. (2004; also Hawley \& Balbus 2002). In simulations of accretion onto a rotating BH De Villiers et al. (2005) find that both gas pressure gradients and Lorentz forces in the inner torus play a significant role in launching the jets.

A confining external pressure is required at the edge of accelerated jets (Komissarov et al. 2007). Therefore, whether a model is based on large scale magnetic fields or not, a confining external medium is required to form a collimated jet, and there is no advantage to magnetic fields-based models. The magnetic fields can further collimate the internal region, i.e., a self collimation (Komissarov et al. 2007).

In Soker \& Regev (2003, hereafter SR03) and Soker \& Lasota (2004, hereafter SL04) we reexamined the launching of jets from accretion disks by thermal pressure following Torbett (1984; also Torbett \& Gilden 1992). This paper describes the basic ingredients of the processes described in those papers, as well as new ideas. 


\section{Motivation}

There are several arguments that point to problems with models for launching jets that are based only on large scale magnetic fields.

(1) Precessing jets. In several YSOs the jets precess on a time scale $\lesssim 100$ years (see e.g. Barsony's poster at this conference). A large scale magnetic field cannot change its symmetry axis on such a short time.

(2) A collimated jets in a planetary nebulae There is a highly collimated clumpy doublejet in the planetary nebulae Hen 2-90 (Sahai \& Nyman 2000), very similar in properties to jets from YSOs that form $\mathrm{HH}$ objects. The source of the accreted mass in planetary nebulae is thought to be a companion star. In such a system large scale magnetic fields are not expected.

(3) No jets in DQ Her (intermediate polars) systems. Intermediate polars (DQ Her systems) are cataclysmic variables where the magnetic field of the accreting WD is thought to truncate the accretion disk in its inner boundary. This magnetic field geometry is the basis for some jet-launching models in YSOs (e.g., Shu et al. 1991). However, no jets are observed in intermediate polars.

(4) Thermal pressure. Thermal pressure seems to be an important ingredient even in MHD models for jet launching (e.g., Ferreira \& Casse 2004; Vlahakis et al. 2003; Vlahakis \& Königl 2003). In particular I note that in the exact solutions for steady relativistic ideal MHD outflows found by Vlahakis \& Königl (2003; also Vlahakis et al. 2003) the initial acceleration phase is by thermal pressure, and internal (thermal) energy is converted to magnetic energy.

\section{Launching jets by thermal pressure gradients}

In YSOs the scheme was developed by Torbett (1984; also Torbett \& Gilden 1992), and discussed in more detail by SR03. SL04 further discussed it, and include accretion into white dwarfs (WDs) as well. In this model, the accreted disk material is strongly shocked due to large gradients of physical quantities in the boundary layer, and then radiatively cools on a time scale longer than the ejection time from the disk.

The model assumes that hundreds of small blobs are formed in the sheared boundary layer (section 2 of SR03). The blobs occasionally collide with each other, and create shocks which cause the shocked regions to expand in all directions. If the shocked regions continue to expand out into the path of yet more circulating blobs, stronger shocks may be created, as was proposed by Pringle \& Savonije (1979) to explain the emission of X-rays out of disk boundary layers in dwarf novae. For the shocked blobs to expand, the radiative cooling time of individual blobs must be longer than the adiabatic expansion time of individual blobs. SR03 demand also that the blobs be small, because the dissipation time of disk material to form the strong shocks must be shorter than the jet ejection time (eq. 24 of SR03). SR03 find that the thermal acceleration mechanism works only when the accretion rate in YSO accretion disks is large enough and the $\alpha$ parameter of the disk small enough - otherwise the radiative cooling time is too short and significant ejection does not take place. SR03 term the strong shocks which are formed from the many weakly shocked blobs, 'spatiotemporally localized (but not too small!) accretion shocks', or SPLASHes. Such SPLASHes can be formed by the stochastic behavior of the magnetic fields of the disk itself and of the central object, e.g., as in cases where the inner boundary of the disk is truncated by the stellar magnetic field.

The model then has two conditions. The first condition is that the strongly shocked gas in the boundary layer will cool slowly, such that the thermal pressure will have enough 
time to accelerate the jet's material. The radiative cooling is via photon diffusion. The constraints translate to a condition on the mass accretion rate to be above a minimum value, depending on the accreting objects, e.g., a WD or a main sequence star. The second condition, which in general is stringent, is that weakly shocked blobs in the boundary layer will expand, and disturb the boundary layer in such a way that a strong shock will develop. This also leads to a minimum value for the mass accretion rate (SR03; SL04 eq. 12)

$$
\dot{M} \gtrsim 4.2 \times 10^{-5} \kappa^{-1}\left(\frac{\alpha}{0.1}\right)\left(\frac{R}{R_{\odot}}\right) M_{\odot} \mathrm{yr}^{-1},
$$

where $R$ is the disk radius from where the jet is launched, $\alpha$ is the disk's viscosity parameter, and $\kappa$ is the opacity. Using this criterion SL04 find that the mass accretion rate above which jets could be blown from accretion disks around YSOs, where $R \simeq R_{\odot}$ and after substituting the opacity, is

$$
\dot{M}_{b}(\mathrm{YSOs}) \gtrsim 7 \times 10^{-7}\left(\frac{R}{R_{\odot}}\right)^{1.2}\left(\frac{\epsilon}{0.1}\right)^{1.4}\left(\frac{\alpha}{0.1}\right) M_{\odot} \mathrm{yr}^{-1},
$$

where $\epsilon=H / R$, and $H$ is the vertical disk's scale height. This condition is drawn in the right hand side of Figure 1 for two sets of $(\alpha ; \epsilon)$ parameters.

For WDs the post shock temperature is much higher than that YSOs, and the opacity is $\kappa=0.4 \mathrm{~cm}^{2} \mathrm{~g}^{-1}$. This gives

$$
\dot{M}_{b}(\mathrm{WD}) \gtrsim 10^{-6}\left(\frac{R}{0.01 R_{\odot}}\right)\left(\frac{\alpha}{0.1}\right) M_{\odot} \mathrm{yr}^{-1} .
$$

This condition is drawn in the left hand side of Figure 1. SL04 noted that this limit is almost never satisfied in CVs (Warner 1995). However, Retter (2004) suggested that this limit might be met during the transition phase in novae, where a claim for a jet has been made.

\section{Jets from brown dwarfs}

The thermal launching model presented here can work only when mass accretion rate is high enough. I therefore turn to check the situation with brown dwarfs (BDs), where the claimed low mass accretion rate was presented as an evidence against the thermallaunching model.

Whelan et al. $(2005,2007)$ argued for a BD jet similar to that observed in YSOs. They use a forbidden line which forms at a critical density of $2 \times 10^{6} \mathrm{~cm}^{-3}$, or $\rho_{c} \simeq$ $3 \times 10^{-18} \mathrm{~g} \mathrm{~cm}^{-3}$, achieved at a distance $r_{o}$. Assuming that the half opening angle of each jet is $\alpha \sim 10^{\circ}$, and the jets speed is $v_{j}$, the outflow rate of the two jets combined is

$$
2 \dot{M}_{j}=4 \pi \rho_{c} r_{o}^{2}(1-\cos \alpha) v_{j} \simeq 8 \times 10^{-10}\left(\frac{r_{o}}{10 \mathrm{AU}}\right)^{2}\left(\frac{\alpha}{10^{\circ}}\right)^{2}\left(\frac{v_{j}}{40 \mathrm{~km} \mathrm{~s}^{-1}}\right) M_{\odot} \mathrm{yr}^{-1}
$$

I substitute numbers as given by Whelan et al. (2007, Table 1) for two systems. For 2MASS1207-3932 I take for the observed values $v_{j}=8 \mathrm{~km} \mathrm{~s}^{-1}$ and $r_{o}=4$ AU, while for $\rho$-Oph $102 \mathrm{I}$ take $v_{j}=40 \mathrm{~km} \mathrm{~s}^{-1}$ and $r_{o}=10$ AU. The unknown inclination implies that both the distance and velocity are larger than the observed values, and the mass outflow rate should be multiply by a number $>2.6$; I multiply it by 2.6 .

For 2 MASS1207-3932 I find $\dot{M}(2 M A S S 1207-3932) \simeq 6.6 \times 10^{-11} M_{\odot} \mathrm{yr}^{-1}$. From Figure 2 of Whelan et al. (2007) it seems that the blue-shifted outflow has a large opening angle (or large covering factor), and I expect that for this case $\alpha>10^{\circ}$. Over all, the 


\section{Accretion into WDs and YSOs}

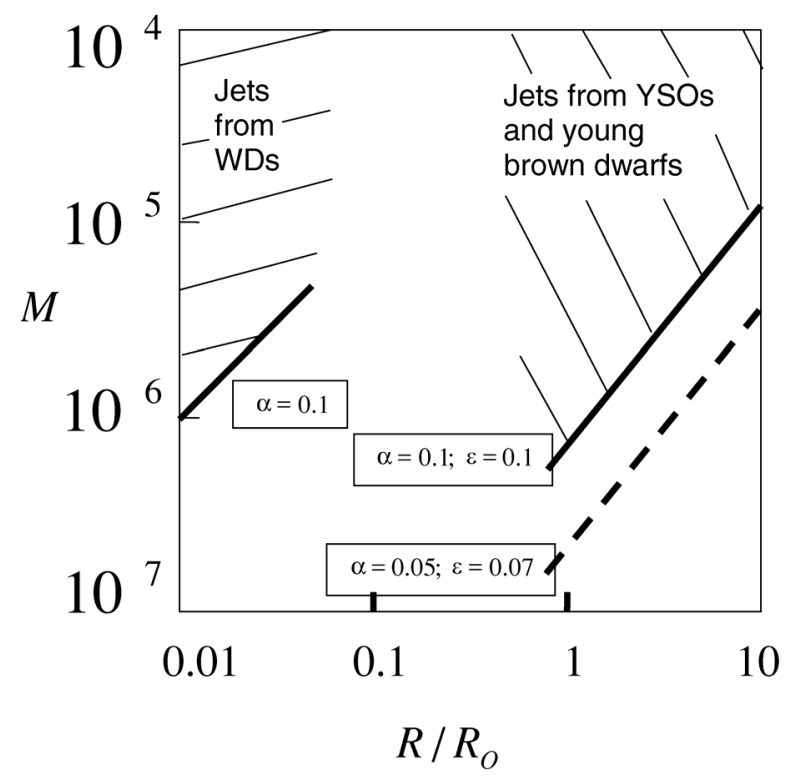

Figure 1. The condition for the radiative cooling time to be longer than mass ejection time, in the accretion rate (in $M_{\odot} \mathrm{yr}^{-1}$ ) versus jet launching radius (in $R_{\odot}$ ) plane. Due to several uncertainties, the allowed mass accretions rate can be an order of magnitude lower, but the general constraints are as shown. The radiative cooling time of the postshock gas is dictated by the photon diffusion time (SR03).

mass loss rate is $\dot{M}(2 M A S S 1207-3932) \gtrsim 10^{-10} M_{\odot} \mathrm{yr}^{-1}$. This is an order of magnitude larger than the accretion rate given in Table 1 of Whelan et al. (2007). For $\rho-\mathrm{Oph} 102$ I find $\dot{M}(\rho-$ Oph 102$) \simeq 2 \times 10^{-9} M_{\odot} \mathrm{yr}^{-1}$. This mass outflow rate is $60 \%$ higher than the mass accretion rate given in Table 1 of Whelan et al. (2007). Jets with opening angle much smaller that $10^{\circ}$ are not likely close to their source in YSOs. Considering that mass outflow rates in YSOs are $0.01-0.1$ times the mass accretion rate, I conclude that there is inconsistency in the data given by Whelan et al. $(2005,2007)$ for outflow from BDs. The resolution to this problem can be one of the following.

(1) The accretion rate is indeed very low $\left(\sim 10^{-11} M_{\odot} \mathrm{yr}^{-1}\right)$, and the mass outflow rate is much smaller than what I estimated above. In that case, the outflow rate $\left(\sim 10^{-12} M_{\odot} \mathrm{yr}^{-1}\right)$ can be accounted for by a BD stellar-type wind, with no need for jets.

(2) The mass accretion rate is much higher than that given by Whelan et al. (2007) and Mohanty et al. (2005), i.e., it is $\dot{M}_{\text {acc }} \simeq 10^{-8} M_{\odot} \mathrm{yr}^{-1}$. In that case jets can be launched according to the model presented here. A higher mass accretion rate is suggested also by the young age of accreting BDs, which is similar to that of YSOs (Mohanty et al. 2005). According to Mohanty et al. (2005) the number of accreting BDs declines substantially by the age of $\sim 10^{7} \mathrm{yr}$. For a BD mass of $M_{\mathrm{BD}}>0.01 M_{\odot}$ the implied average accretion rate should be $\gtrsim 10^{-9} M_{\odot} \mathrm{yr}^{-1}$. A short accretion phase for YSOs is also suggested by the work of Lucas Cieza et al (2007).

(3) There are very large variations on short time scales of the mass accretion rate, as suggested by Scholz \& Jayawardhana (2006). Jets are launched then only during the 

Single Stage Double Stage
Acceleration Acceleration

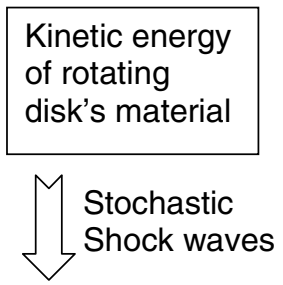

Internal
(e.g., thermal)
energy

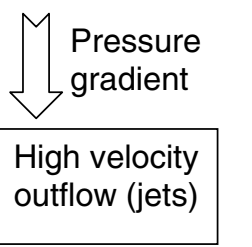

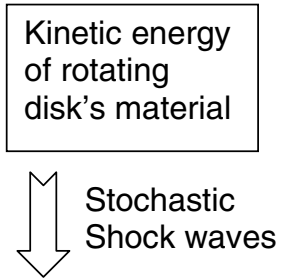

$$
\begin{aligned}
& \text { Internal } \\
& \text { (e.g., thermal) } \\
& \text { energy }
\end{aligned}
$$

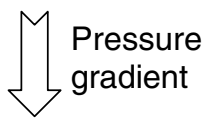

\section{Outflow}
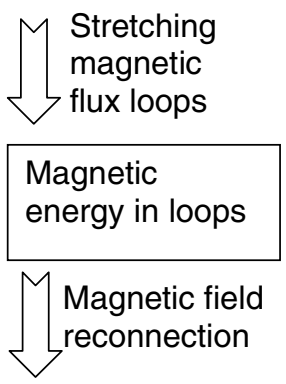

$$
\begin{aligned}
& \text { High velocity } \\
& \text { outflow (jets) }
\end{aligned}
$$

\section{Solar}

Flares

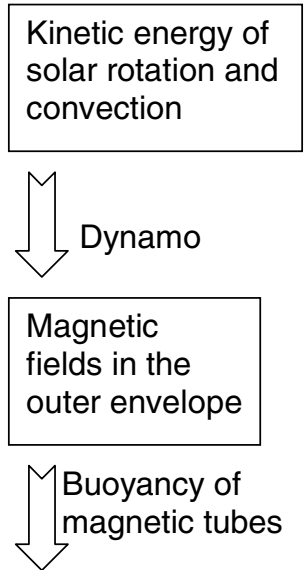

\section{Outflow}

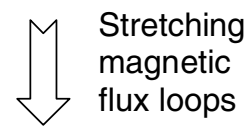

$$
\begin{array}{|l|}
\hline \begin{array}{l}
\text { Magnetic } \\
\text { energy in loops }
\end{array} \\
M \begin{array}{l}
\text { Magnetic field } \\
\text { reconnection }
\end{array}
\end{array}
$$

High velocity ejection

Figure 2. The energy transformations in the single-stage acceleration scenario and in the double-stage acceleration scenario.

very high mass accretion phases. On average, the mass accretion rate can stay low. The outflow is composed of many small clumps, and the average mass outflow rate is much smaller than what I calculated above.

I predict that future studies will show that the mass accretion rate of BDs that launch jets (and not stellar-type winds) is $\dot{M}_{\text {acc }}(B D s) \gtrsim 10^{-9}-10^{-8} M_{\odot} \mathrm{yr}^{-1}$, i.e., larger than current studies show (e.g., Mohanty et al. 2005). Such an accretion rate with a low enough value of the disk-viscosity parameter $\alpha$ (see equation 3.2), is compatible with the model presented here.

\section{A double-stage acceleration scenario}

I propose the possibility that thermal energy is transfered to magnetic energy, via kinetic energy of the outflowing gas, and a second stage of acceleration takes place wherein 
magnetic field reconnection accelerates the gas to velocities much above the escape velocity, as in solar flares. The energy cycle is as depicted in Figure 2.

The idea that magnetic energy can serve as an intermediate stage is not new. In launching jets from black holes and neutron stars the dissipated bulk kinetic energy might be channelled to magnetic energy, and then to kinetic energy. In the exact solutions for steady, relativistic, ideal MHD outflows found by Vlahakis \& Königl (2003; also Vlahakis et al. 2003) the initial acceleration phase is by thermal pressure, and internal (thermal) energy is converted to magnetic energy. I consider a non-steady state outflow, based on flare-like ejection, as in solar coronal mass ejection (CME), rather than a steady state outflow. Direct build-up of magnetic fields, without the intermediate stage of thermal energy, is discussed by Machida \& Matsumoto (2003, their $\S 4$ ) who show how in the plunging region of the accretion disk around $\mathrm{BHs}$, where gas falls to the $\mathrm{BH}$ and no stable orbits are possible, the gravitational energy of the accreting gas is converted to magnetic energy.

The conversion of accretion energy (kinetic energy of the rotating disk or gravitational energy) to thermal energy, then to magnetic field, and then to the kinetic energy of the ejected wind will be studied in a future paper.

\section{Acknowledgements}

I thank Oded Regev for many useful comments. This research was supported by the Asher Fund for Space Research at the Technion.

\section{References}

De Villiers, J. P., Hawley, J. F., \& Krolik, J. H. 2004, ApJ, 599, 1238

De Villiers, J. P., Hawley, J. F., Krolik, J. H., \& Hirose, S. 2005, 620, 878

Ferreira, J. 2002, in Star Formation and the Physics of Young Stars - Summer school on Stellar Physics X - EAS Vol. III, ed. J. Bouvier \& J.-P. Zahn (EDP Books)

Ferreira, J., \& Casse, F. 2004, ApJ, 601, L139

Ferreira, J. \& Pelletier, G. 1993, A\&A, 276, 625

Ferreira, J. \& Pelletier, G. 1995, A\&A, 295, 807

Hawley, J. F., \& Balbus, S. A. 2002, ApJ, 573, 738

Komissarov, S. S., Barkov, M. V., Vlahakis, N., Königl, A. 2007. MNRAS, in press (arXiv:astro$\mathrm{ph} / 0703146)$

Königl, A., \& Pudritz, R.E. 2000, in Protostars and Planets IV, ed. V. Mannings, A. P. Boss \& S. S. Russell, (Univ. of Arizona Press, Tucson), 759

Krasnopolsky, R., Li, Z.-Y., \& Blandford, R. D. 2003, ApJ, 595, 631

Lucas Cieza et al. 2007, ApJ in press (arXiv:0706.0563

Machida, M., \& Matsumoto, R. 2003, ApJ, 585, 429

Mohanty, S., Jayawardhana, R., \& Basri, G. 2005, ApJ, 626, 498

Pringle, J. E. \& Savonije, G.J. 1979, MNRAS, 187, 777

Retter, A. 2004, ApJ, 615, L125

Sahai, R. \& Nyman, L.-A. 2000, ApJ 538, L145

Scholz, A., \& Jayawardhana, R. 2006, ApJ, 638, 1056

Shu, F. H., Lizano, S., Ruden, S., \& Najita, J. 1988, ApJ, 328, L19

Shu, F.H., Najita, J.R., Shang, H. \& Li Z.-Y. 2000, in Protostars and Planets IV, ed. V. Mannings, A. P. Boss \& S. S. Russell, (Univ. of Arizona Press, Tucson), 789

Shu, F. H., Ruden, S.P., Lada, C.J. \& Lizano, S. 1991, ApJ, 370, L31

Soker, N., \& Lasota, J.-P. 2004, A\&A, 422, 1039 (SL04)

Soker, N., \& Regev, O. 2003, A\&A, 406, 603 (SR03)

Torbett, M. V. 1984, ApJ, 278, 318

Torbett, M. V., \& Gilden, D. L. 1992, A\&A, 256, 686 (TG) 
Vlahakis, N., \& Königl, A. 2003, ApJ, 596, 1104

Vlahakis, N., Fang, P., \& Königl, A. 2003, ApJ594, L23

Wardle, M., \& Königl, A. 1993, ApJ, 410, 218

Warner, B. 1995, Cambridge Astrophysics Series, Cambridge, New York: Cambridge University Press, 1995,

Whelan, E. T., Ray, T. P., Bacciotti, F., Natta, A., Testi, L., \& Randich, S. 2005, Natur, 435, 652

Whelan, E. T., Ray, T. P., Randich, S., Bacciotti, F., Jayawardhana, R, Testi, L., Natta, A., \& Mohanty, S. 2007, ApJ, 659, L45

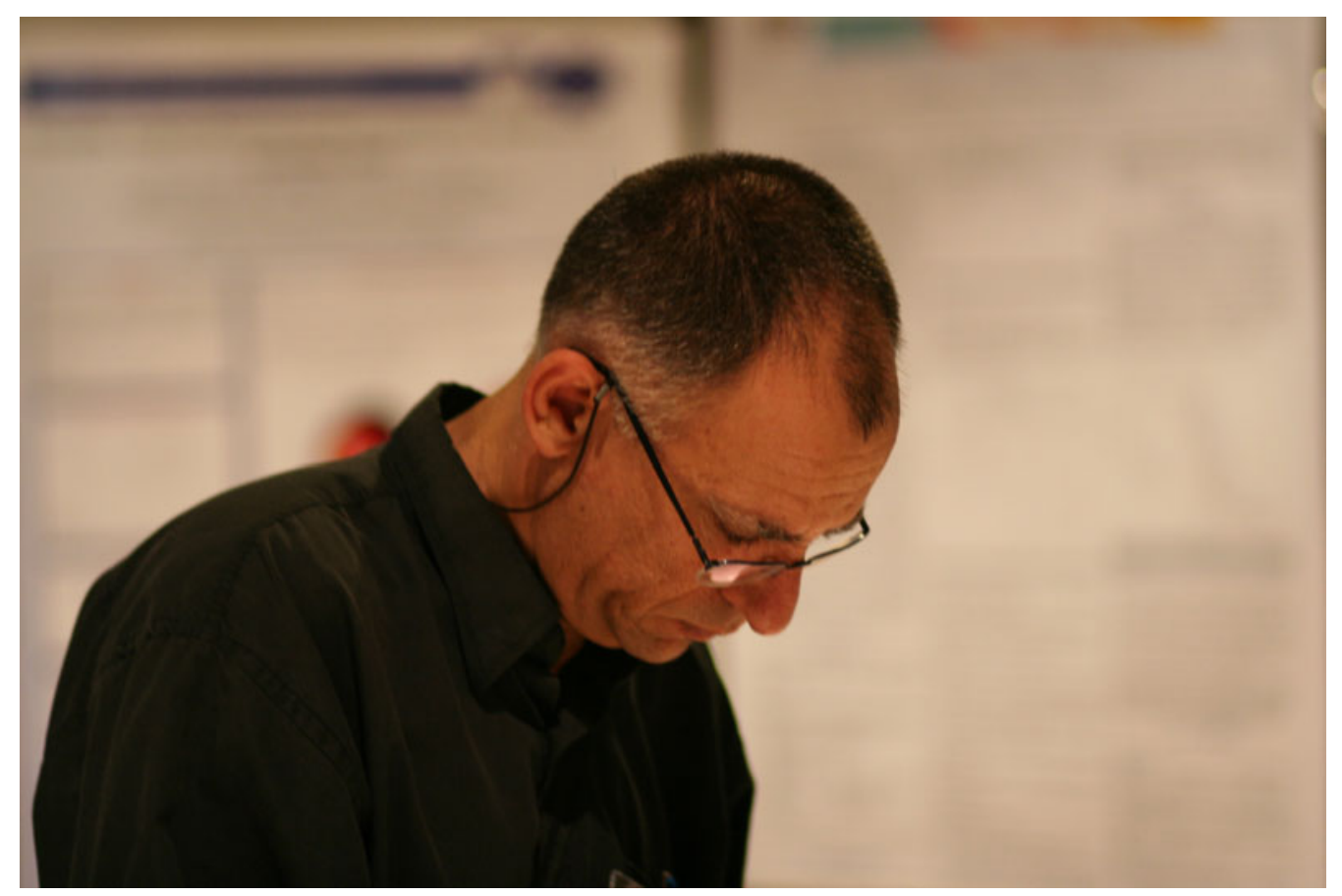



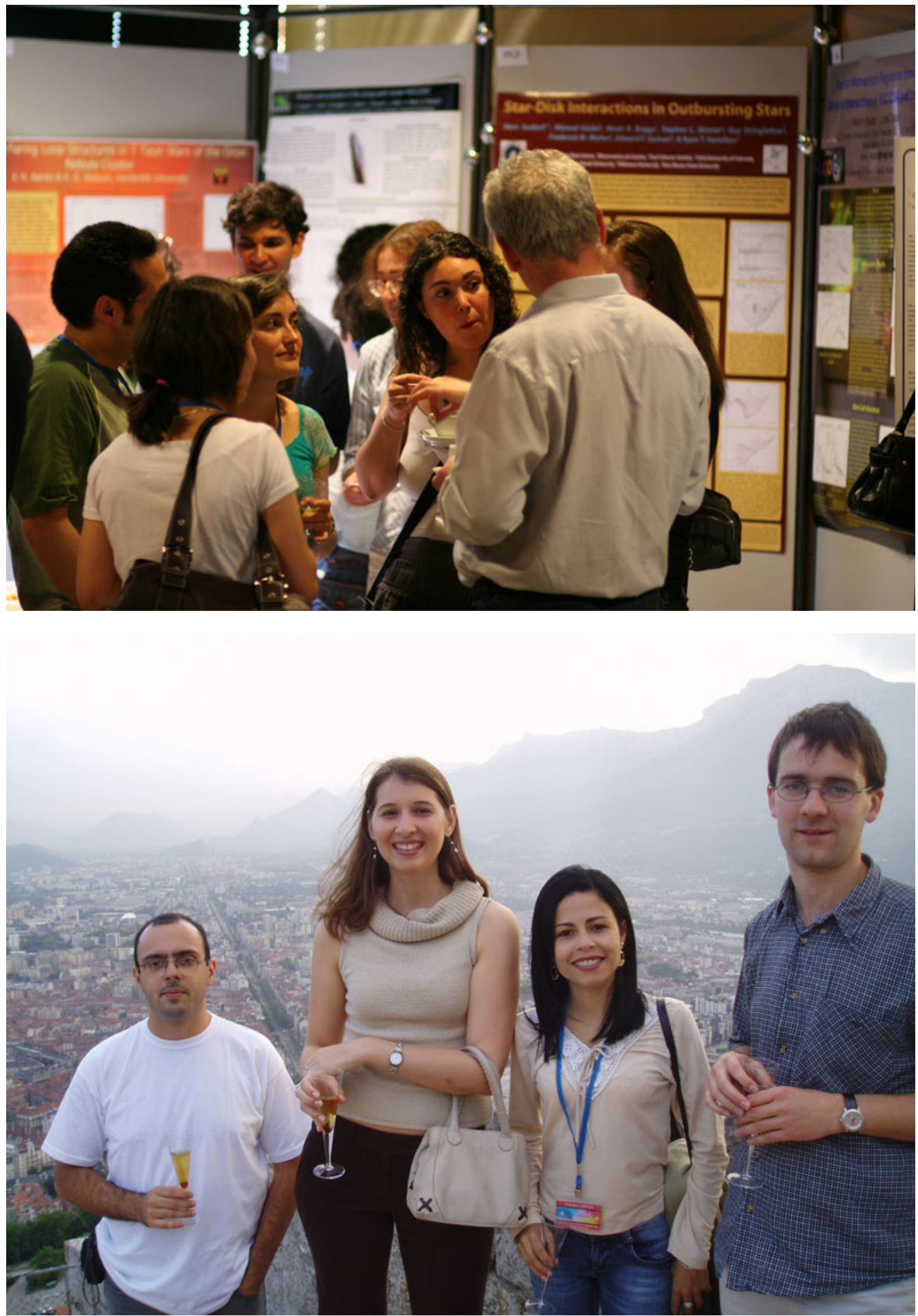